\title{
Epidermal HLA-DR and the enhancement of cutaneous reactivity to superantigenic toxins in psoriasis
}

\author{
Jeffrey B. Travers, ${ }^{1}$ Qutayba A. Hamid, ${ }^{2}$ David A. Norris, ${ }^{3}$ Christine Kuhn, ${ }^{1}$ \\ Ralph C. Giorno, ${ }^{3}$ Patrick M. Schlievert, ${ }^{4}$ Evan R. Farmer, ${ }^{1}$ and Donald Y.M. Leung 3,5
} ${ }^{1}$ Departments of Dermatology and Pharmacology, Indiana University Medical Center, Indianapolis, Indiana 46202, USA
${ }^{2}$ Meakins-Christie Laboratories and Departments of Pathology, McGill University, Montreal, Quebec H2X 2P2, Canada
${ }^{3}$ Departments of Pediatrics, Dermatology, and Medicine, University of Colorado Health Sciences Center,
Denver, Colorado 80262, USA
${ }^{4}$ Department of Microbiology, University of Minnesota, Minneapolis, Minnesota 55455, USA
${ }^{5}$ Division of Pediatric Allergy and Immunology, The National Jewish Medical and Research Center,
Denver, Colorado 80206, USA

Address correspondence to: Donald Y.M. Leung, The National Jewish Medical and Research Center, 1400 Jackson Street, Room K926, Denver, Colorado 80206, USA.

Phone: (303) 398-1379; Fax: (303) 270-2182; E-mail: leungd@njc.org.

Received for publication March 16, 1999, and accepted in revised form September 10, 1999.

Streptococcal and staphylococcal superantigens (SAg's) have been implicated in the pathogenesis of inflammatory skin diseases, but the mechanisms by which these toxins act are unknown. The present study assessed the ability of nanogram quantities of topically applied purified toxic shock syndrome toxin-1 (TSST-1), staphylococcal enterotoxin type B, and streptococcal pyrogenic enterotoxin types $\mathrm{A}$ and $\mathrm{C}$ to induce inflammatory reactions in clinically uninvolved skin of normal controls and subjects with psoriasis, atopic dermatitis, and lichen planus. These SAg's triggered a significantly greater inflammatory skin response in psoriatics than in normal control subjects or in subjects with atopic dermatitis or lichen planus. Surprisingly, skin biopsies did not exhibit the T-cell receptor V $\beta$ stimulatory properties predicted for SAg-induced skin reactions. By 6 hours after patch testing with SAg's, TNF- $\alpha$ mRNA had increased in the epidermis (but not the dermis) in biopsies from psoriatics, compared with controls. Immunohistochemical studies revealed significantly higher HLA-DR expression in keratinocytes from psoriatics than from controls. However, a mutant TSST-1 protein that fails to bind HLA-DR did not elicit an inflammatory skin reaction. These results indicate that keratinocyte expression of HLA-DR enhances inflammatory skin responses to SAg's. They may also account for previous studies failing to demonstrate selective expansion of T-cell receptor V $\beta s$ in psoriatics colonized with SAg-producing Staphylococcus aureus, and they identify a novel T cell-independent mechanism by which SAg's contribute to the pathogenesis of inflammatory skin diseases.

J. Clin. Invest. 104:1181-1189 (1999).

\section{Introduction}

Psoriasis is a chronic inflammatory skin disorder affecting $1-2 \%$ of the general population. The characteristic lesion of psoriasis is a sharply demarcated erythematous papule or plaque containing hyperproliferating keratinocytes as well as infiltrating neutrophils, monocytes, and T lymphocytes (1). Although psoriasis is considered an autoimmune disease, increasing evidence suggests an important role for bacteria in its initiation and/or propagation. Colonization and infection with Staphylococcus and Streptococcus have been reported to exacerbate psoriasis $(2,3)$. In this regard, Staphylococcus aureus has been found on the skin of more than half the patients with chronic plaque psoriasis (2). We have previously identified patients with psoriasis vulgaris who have experienced exacerbations of their disease in association with staphylococcal skin infections (4). The most convincing clinical and experimental association between bacterial infection and psoriasis, however, is in patients with acute guttate (eruptive) psoriasis $(3,5)$.
Given the strong association between bacterial infection and psoriasis, intensive studies have sought to discern the mechanisms by which bacteria participate in the pathogenesis of this common skin disease. Recent studies have demonstrated that streptococcal pyrogenic exotoxins (SPEs) and staphylococcal enterotoxins can act as superantigens $(6,7)$, providing plausible mechanisms by which these bacteria could cause an inflammatory skin lesion containing activated $\mathrm{T}$ cells and monocytes. The term superantigen (SAg) was coined to describe a family of microbial proteins that are potent stimulators of $\mathrm{T}$ cells and macrophages $(6,7)$. When bound to MHC class II molecules, SAg's stimulate T cells expressing particular T-cell receptor (TCR) V $\beta$ gene segments (8). In addition to this ability to activate large numbers of T cells, in vitro studies have reported that SAg's can also activate and stimulate cytokine production from MHC class II-expressing cells, including activated keratinocytes (9-11), independent of T cells. The latter effect is transduced through the MHC class II molecule (11). 
The association between SAg's and psoriasis has been strengthened by recent reports culturing streptococcal pyrogenic exotoxin serotype C-producing (SPEC- or scarlet fever type C-producing) group A streptococcus from the oropharynx of patients with acute guttate psoriasis and demonstrating increased numbers of $\mathrm{V} \beta 2$ expressing $T$ cells in their lesional skin $(5,12)$. In addition, recent studies from 2 separate groups of investigators indicate that normal-appearing skin from psoriatic patients grafted onto immunodeficient mice can be induced to develop into psoriatic lesions by repeated injection with autologous SAg-treated immunocytes $(13,14)$. Together, these findings suggest that SAg stimulation can initiate psoriasis. To date, however, there have been no in vivo studies in humans directly examining the effects of SAg's on the uninvolved skin of psoriatic patients. The objective of the present study was to evaluate the reactivity of psoriatic skin to topically applied bacterial SAg's and determine the mechanisms by which they induce skin inflammation in vivo in psoriasis.

\section{Methods}

Patients. Fifty-seven adult patients were enrolled into this study. Twenty-six patients with type I (15) psoriasis (age range, $23-52$ years; mean, 35 years); 6 patients with atopic dermatitis (age range, 21-28; mean, 25 years), diagnosed according to the Hanifin and Rajka criteria (16); and 5 patients with biopsy-proven lichen planus (age range, $24-56$ years; mean, 43 years) also participated in this study. Twenty-one subjects (age range, 22-52 years; mean, 32 years) without a personal or family history of skin disease or respiratory allergy were enrolled into the study to serve as normal controls. Patients refrained from using topical medications to the arm undergoing patch testing and from using oral antihistamines for at least 2 weeks before patch testing. None of the patients was on any systemic immunosuppressive drugs, including corticosteroids or cyclosporin. The protocols involving human subjects were approved by the institutional review boards of both the University of Colorado Health Sciences Center and the Indiana University School of Medicine. Informed consent was obtained from all subjects before performing all studies.
Patch-testing protocol. Staphylococcal and streptococcal exotoxins were purified by Patrick M. Schlievert as described previously (17). In selected experiments, a mutant toxic shock syndrome toxin-1 (TSST-1) protein, G31S/S32P, produced by site-directed mutagenesis was also used. G31S/S32P has previously been demonstrated to lack HLA-DR binding $(18,19)$.

A total of $20 \mu \mathrm{L}$ of $1 \mu \mathrm{g} / \mathrm{mL}$ solutions of highly purified TSST-1, staphylococcal enterotoxin B (SEB), streptococcal pyrogenic exotoxins A and C (SPEA, SPEC) or G31S/S32P in PBS, or PBS alone were placed in 8- $\mathrm{mm}$ diameter Finn chambers (Epitest Ltd., Oy, Finland) and applied to uninvolved skin on the volar forearm. Most experiments used tape-stripping a $2 \times 12 \mathrm{~cm}$ section of uninvolved skin 100 times with cellophane tape $(3 \mathrm{M}$ Inc., Minneapolis, Minnesota, USA) before application of the patches. Twenty microliters of $1 \%(\mathrm{vol} / \mathrm{vol})$ sodium lauryl sulfate (Sigma Chemical Co., St. Louis, Missouri, USA) was used as an irritant control. At 48 hours, the patch tests were removed and clinically assessed. The following clinical measurement system was used to rate the reactions: $0=$ no reaction; $1=$ nonpalpable macular erythema; 2 = palpable erythema; and $3=$ palpable erythema extending beyond the test chamber. After clinical assessment, 4-mm punch biopsies were obtained from the reaction sites, with part fixed in buffered formalin, routinely processed for $4-\mu \mathrm{m}$ paraffin-embedded sections, and stained with hematoxylin/eosin. The other half was snap-frozen in OCT for immunohistochemical studies.

Quantitation of skin inflammatory cell infiltrate. To quantitate mononuclear inflammatory cells in the dermis of biopsy specimens, an eyepiece counting grid (Olympus Optical Co. Tokyo, Japan) was used at $\times 200$, which provided an area of $0.25 \mathrm{~mm}^{2}$. The grid was placed at the left side of the tissue with the top of the grid touching the dermal-epidermal junction. All the mononuclear cells in the grid were counted. Three adjacent $0.25-\mathrm{mm}^{2}$ squares were counted lateral to the first to assay a total area of $1.0 \mathrm{~mm}^{2}$ encompassing the papillary and part of the reticular dermis. Similarly, the grid was placed at the right side of the tissue, and 3 more sections were counted. The counts were averaged and then multiplied by 4 to give the number of mononuclear cells per square millimeter. Duplicate

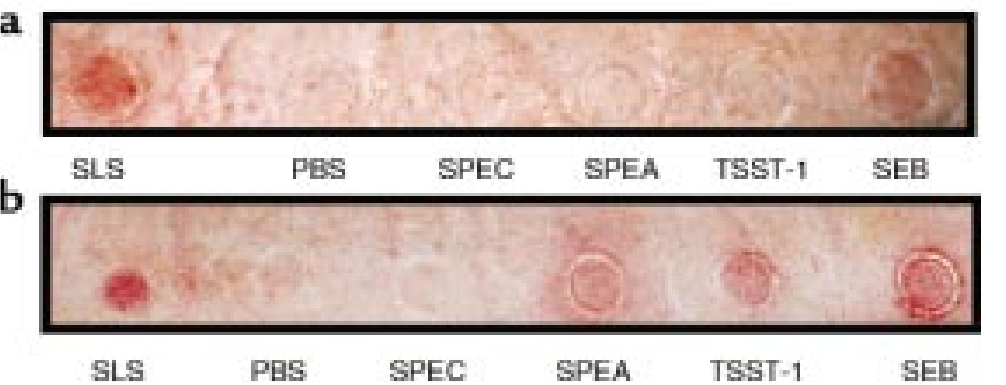

Figure 1

Typical examples of 48 hours of closed patch testing of exotoxins, PBS vehicle, and SLS on a normal subject (a) and a patient with psoriasis (b). Subjects underwent tape-stripping followed by closed patch testing of exotoxins SEB, TSST-1, SPEA, SPEC, PBS vehicle, or irritant SLS for 48 hours, as outlined in Methods. After removal of patches, the reactions were clinically rated as follows: $0=$ no reaction; $1+=$ macular erythema; $2+=$ palpable erythema; $3+=$ palpable erythema extending outside of the patch test site. These reactions were graded as follows: (a) SLS $1+$, PBS 0, SPEC 0, SPEA 0, TSST-1 0, SEB 1+; (b) SLS 1+, PBS 0, SPEC 0, SPEA 3+, TSST-1 1+, SEB 2+. 
Table 1

Mononuclear cell count and clinical reactivity to SEB application in controls and psoriasis subjects

\begin{tabular}{cccccc}
\hline $\begin{array}{c}\text { Psoriatic } \\
\text { subject }\end{array}$ & $\begin{array}{c}\text { Mononuclear } \\
\text { cells } / \mathrm{mm}^{2}\end{array}$ & $\begin{array}{c}\text { Clinical } \\
\text { rating }\end{array}$ & $\begin{array}{c}\text { Normal } \\
\text { subject }\end{array}$ & $\begin{array}{c}\text { Mononuclear } \\
\text { cells/mm }\end{array}$ & $\begin{array}{c}\text { Clinical } \\
\text { rating }\end{array}$ \\
P-1 & 1,509 & $2+$ & $\mathrm{N}-1$ & 422 & $1+$ \\
P-2 & 1,123 & $2+$ & $\mathrm{N}-2$ & 108 & $1+$ \\
P-3 & 1,137 & $2+$ & $\mathrm{N}-3$ & 1,089 & $1+$ \\
P-4 & 2,097 & $2+$ & $\mathrm{N}-4$ & 345 & $1+$ \\
P-5 & 1,761 & $3+$ & $\mathrm{N}-5$ & 147 & $1+$ \\
P-6 & 2,372 & $2+$ & $\mathrm{N}-6$ & 171 & $1+$ \\
P-7 & 270 & $1+$ & & & \\
P-8 & 1,455 & $1+$ & & &
\end{tabular}

Eight psoriatic and 6 control subjects who underwent tape-stripping followed by closed patch testing with exotoxins, PBS, and SLS underwent biopsies of the SEB reactions at 48 hours. The data listed are the mean number of mononuclear cells per square millimeter of tissue obtained as outlined in Methods, along with the clinical reaction (graded as outlined in Figure 2).

USA), anti-CD3 $\mathrm{mAb}$, or mouse immunoglobulin isotype control (Becton Dickinson Immunocytometry Systems, San Jose, California, USA). Sections were then washed and stained by the labeled avidin biotin method using peroxidaselabeled streptavidin (DAKO) as described previously (5). Afterward, sections were counterstained with hematoxylin and mounted. Control antibodies were tested on step sections of the same tissue specimens. The negative staining controls were isotype-specific mouse immunoglobulins with irrelevant specificity. Normal human tonsil sections were used as positive controls for $\mathrm{V} \beta \mathrm{T}$ cell staining and CD antibodies.

Quantitation and statistical analysis. The

slides from each biopsy were counted and averaged. All cell counts were done by one of the authors (E.R. Farmer) under blinded conditions.

In situ hybridization studies. In these experiments, skin biopsy specimens were fixed immediately in freshly prepared 4\% paraformaldehyde/PBS solution for 2 hours, washed in 15\% sucrose/PBS 3 times, embedded in OCT compound (Tissue-Tek; Miles Inc., Elkhart, Indiana, USA), and snap-frozen in isopentane cooled in liquid nitrogen. Cryostat sections $5 \mu \mathrm{m}$ thick were cut on $0.1 \%$ poly-L-lysine-coated slides, air-dried overnight at $37^{\circ} \mathrm{C}$, and stored at $-80^{\circ} \mathrm{C}$ until used. In situ hybridization studies for IL-4, IFN- $\gamma$, and TNF- $\alpha$ mRNA were performed as described previously using digoxigeninlabeled cRNA probes $(20,21)$. The following control experiments were also performed: (a) omitting the probe in the in situ hybridization protocol and using a sense dig-labeled riboprobe; (b) pretreatment of slides with RNase A before in situ hybridization; (c) use of an unrelated antisense digoxigenin-labeled RNA probe (human atrial natriuretic polypeptide); and (d) omitting the digoxigenin-alkaline-phosphatase conjugate.

Immunohistochemistry studies. Cryostat sections prepared as described for in situ hybridization studies were used for analysis of HLA-DR expression. Immunoreactivity was assessed by using the alkaline phosphatase antialkaline phosphatase technique and by using either an anti-HLA-DR, anti-CD25, anti-CD4, or anti-CD8 $\mathrm{mAb}$ (DAKO Canada Inc.; Mississauga, Ontario, Canada) as described previously (22). The reaction was viewed with fast red alkaline-phosphatase substrate. As controls, sections were processed in the absence of the primary antibody. System and specificity controls were also included in each staining run, using human tonsils, obtained at routine tonsillectomy operations, and mouse IgG2a myeloma proteins as a negative control.

For studies analyzing TCR V $\beta$ expression, $4-\mu \mathrm{m}$ cryostat sections were cut, dehydrated in acetone for $10 \mathrm{~min}$ utes, and air-dried. Sections were incubated 30-60 minutes at room temperature with the respective mouse anti-human $\mathrm{V} \beta \mathrm{mAb}$ (Immunotech, Westbrook, Maine, readings of all histological, immunohistochemical, and in situ hybridization studies were conducted in blinded fashion without knowledge of the subject's identity or disease condition. Epidermal TNF- $\alpha$ mRNA and HLADR immunoreactivity was expressed as percentage of epidermal cells that express TNF- $\alpha$ mRNA or HLA-DR protein. Subepidermal mRNA expression of TNF- $\alpha$ and

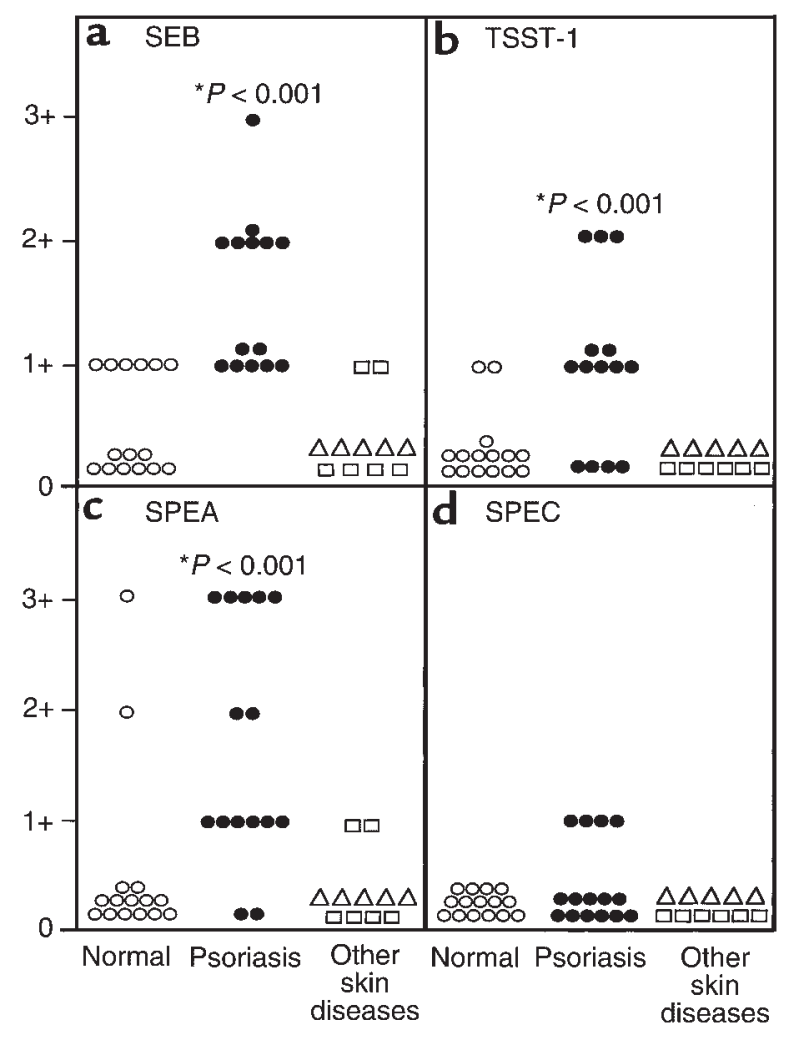

Figure 2

Cutaneous reactivity of exotoxins on tape-stripped skin. Subjects with psoriasis, atopic dermatitis, lichen planus, or normal controls underwent our protocol of epidermal modification followed by closed patch testing. After removal of patches, the reactions were clinically rated as outlined in Figure 1. ${ }^{*} P<0.001$ vs. normal controls. 


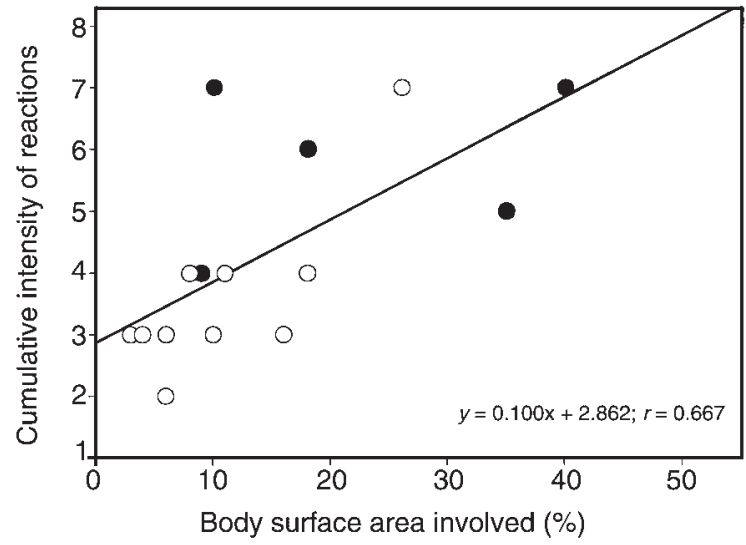

Figure 3

Comparison of the cutaneous reactivity of psoriatics to exotoxins with extent of skin disease. At the time of closed patch testing, the amount of disease activity of psoriatic subjects was measured as percentage of body surface area involvement for the comparison. The cumulative clinical reactions to the 4 exotoxins tested were then compared with the percentage of body surface area involvement of psoriasis. The slope of the resulting curve was found to be statistically different from $0(P<0.05)$. Subjects who were found to develop psoriasis lesions in the sites of their skin biopsies (positive isomorphic phenomenon) are depicted by filled circles.

immunoreactivity for HLA-DR were presented as number of cells per square millimeter. Statistical analyses used the unpaired $t$ test (Instat Program; GraphPad Software for Science Inc., San Diego, California, USA) to assess clinical differences in exotoxin reactivities between subject groups and Wilcoxon rank order test to assess differences between mononuclear cell counts in biopsy specimens from psoriatics and control subjects.

\section{Results}

Closed patch testing of SAg toxins. Initially, 6 normal and 4 psoriatic patients underwent closed patch testing with 20 ng TSST-1, SEB, SPEA, and SPEC on uninvolved volar forearm skin. Forty-eight-hour closed patch testing did not induce cutaneous reactions to any of the toxins, yet all experienced a reaction to the irritant (1\%) SLS. These preliminary studies suggested that a single application of this concentration of toxins to intact skin was not sufficient to elicit an inflammatory skin reaction.

Tape-stripping is a standardized method used to induce epidermal activation and to increase cutaneous absorption by removal of the stratum corneum (23). To determine whether epidermal modification was required for nanogram amounts of SAg to induce skin inflammatory responses, volar forearm skin was treated with tape-stripping before application of the toxins as described in Methods. Tape-stripping was repeated 100 times, as it has been shown by Nickoloff and Naidu that this protocol resulted in the maximal amount of keratinocyte activation in normal skin (23).

Using this protocol of epidermal modification and 48 hours of closed patch testing, positive reactions to the SAg's and SLS were noted. No clinical reactions were seen to the PBS vehicle. The SAg reactions consisted of erythematous macules or plaques and were usually asymptomatic. The SLS reactions consisted of erythematous thin plaques, but unlike the SAginduced reactions, were uniformly associated with mild-moderate pruritus. The reactions to SAg's differed greatly among the subjects, however, even the most vigorous $(3+)$ reactions resolved within 1 week. No systemic symptoms, such as fever, headache, dizziness, or presence/worsening of skin rash, were noted in subjects undergoing these protocols.

Although skin reactions were elicited in both psoriatic and normal subjects, psoriatics reacted to more SAg's and with greater intensity than normal subjects. Examples of reactions seen in normal and psoriatic subjects to the various exotoxins are shown in Figure 1. Figure 2 compares the intensity of clinical reactions exhibited by psoriatic and control subjects. Statistically significant $(P<0.001)$ increased clinical reactions to SEB, TSST-1, and SPEA were seen in psoriatic versus control populations. Only 4 of 15 psoriatic subjects, but 0 of 15 control subjects reacted to SPEC (not significant). Subjects with atopic dermatitis (6 subjects) or the papulosquamous disease lichen planus (5 subjects) reacted in a similar fashion to normal controls (Figure 2). The cutaneous reactivity to exotoxins correlated with the extent of psoriasis (as assessed by percentage of body surface area involvement) as shown in Figure 3. As depicted in Figure 3, 5 of 15 (4 of highest
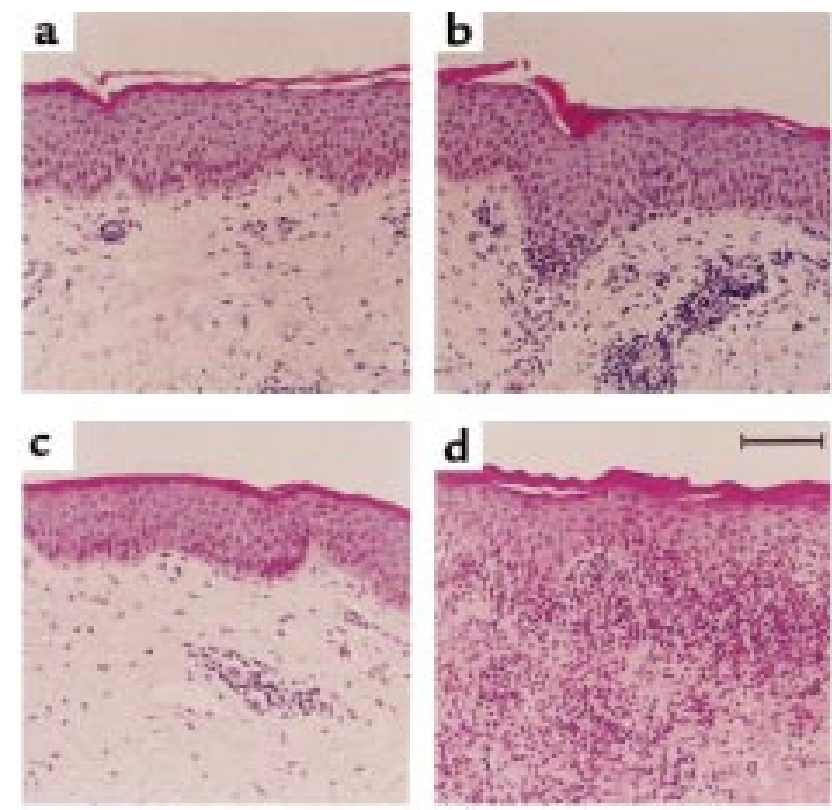

\section{Figure 4}

Histological evaluation of exotoxin-induced cutaneous reactions. Biopsies of reactions from subjects undergoing tape-stripping and closed patch testing for 48 hours were formalin-fixed, processed in paraffin, and stained with hematoxylin and eosin. (a) PBS reaction in a normal subject; (b) $1+$ reaction to SEB in a normal subject; (c) PBS reaction in a psoriatic patient; and (d) $2+$ reaction to SEB in a psoriatic patient. 



Figure 5

Representative examples of TNF- $\alpha$ mRNA in situ hybridization ( $a$ and b) and HLA-DR immunostaining using APAAP technique ( $\boldsymbol{c}$ and $\mathbf{d}$ ). Shown in $\mathbf{a}$ is a representative example of TNF- $\alpha$ mRNA in the epidermis of a skin biopsy from a patient with psoriasis after 6 hours of SEB $(\times 500)$; $\boldsymbol{b}$ is a skin section probed for TNF- $\alpha$ mRNA from a normal subject after 6 hours SEB $(\times 500)$; $c$ is an example of HLA-DR immunoreactivity in the epidermis of a patient with psoriasis after SEB application for 6 hours $(\times 500)$; and $\mathbf{d}$ assesses HLA-DR immunoreactivity in the epidermis of a normal subject after SEB application for 6 hours.

responders) psoriatic subjects developed psoriatic lesions in the skin biopsy sites. Unlike the SAg-induced cutaneous reactions, those induced by SLS were similar among subjects with psoriasis, atopic dermatitis, lichen planus, and normal controls.

Histological evaluation of SAg-induced reactions. At 48 hours when the patches were removed to assess the clinical reactions, skin biopsies were obtained from some psoriatic and normal subjects for both routine histology and immunohistochemistry. As shown in Figure 4, the reactions consisted of epidermal and dermal spongiosis, with mononuclear (lymphocytic and monocytic) cell infiltrates, both in the papillary dermis and the epidermis. Neither eosinophils nor neutrophils were seen in significant numbers in the biopsy specimens. In several of the biopsies taken from psoriatic patients, mild epidermal acanthosis was noted. Intrapapillary thinning or neutrophilic microabscesses, characteristic of psoriasis lesions, were not seen. For positive SEB reactions in 8 psoriatic and the 6 normal subjects, the numbers of inflammatory cells were examined. As shown in Table 1 , statistically significant $(P=$ 0.045 by the Wilcoxon rank order test) increased numbers of mononuclear cells were seen in the psoriatic versus control populations.

Characterization of T cells in SAg-induced reactions. Because selective expansion of TCR V $\beta$ is a hallmark of superantigenic stimulation of $T$ cells $(5,8)$, the reactions were assessed for the presence of TCR V $\beta$ expansion by immunohistochemistry. Staining of skin specimens from 6 subjects with positive (48 hours) exotoxininduced reactions with mAb's directed to TCR V 2 (TSST-1 and SPEC reactive), 3 (SEB reactive), 8.1 (SPEA reactive), 12 (SEB and SPEA reactive), and 17 (SEB reactive) failed to demonstrate selective TCR V $\beta$ expansion that correlated with the predicted stimulatory properties of the SAg (data not shown). These findings are in contrast to our ability to demonstrate marked expansion of $\mathrm{V} \beta 2^{+} \mathrm{T}$ cells in the skin lesions of guttate psoriasis (5).

We also examined the T-cell infiltrate in skin biopsies of SEB- and PBS-stimulated sites from psoriasis patients for CD25 (as a marker of T-cell activation), CD4, and CD8 expression. SEB-stimulated sites compared with PBS-stimulated sites contained increased numbers of $\mathrm{T}$ cells per square millimeter expressing CD25 (54 \pm 15 vs. $10 \pm 2 ; P<0.05), C D 4(160 \pm 10$ vs. 71 $\pm 20 ; P<0.05)$, and CD8 (57 \pm 7 vs. $23 \pm 6 ; P<0.05)$. SEB-stimulated skin sites also expressed increased IFN$\gamma$ mRNA (33 \pm 4 vs. $8 \pm 4$ cells $\left./ \mathrm{mm}^{2} ; P<0.05\right)$ expression, but not increased IL- 4 mRNA ( $11 \pm 2$ vs. $10 \pm 6$ cells $/ \mathrm{mm}^{2} ; P=$ not significant) expression.

TNF- $\alpha m R N A$ levels in SAg-induced reactions. SAg's have been shown to stimulate human keratinocytes (HKs) to produce TNF- $\alpha$ in vitro $(9,10,24)$. To assess the potential role of cytokines in the modulation of in vivo skin inflammatory responses to SAg's, in situ hybridization studies of the cutaneous reactions were conducted. In these experiments, volar forearm skin was tapestripped and SEB or PBS vehicle was applied. At 6 hours, the patches were removed and TNF- $\alpha$ mRNA levels were assessed in the skin biopsies. In situ hybridization studies of unmodified skin from both normal controls and psoriatics did not reveal significant levels of TNF- $\alpha$ mRNA (data not shown). However, as shown in Figures 5 and 6, psoriatic skin expressed significantly higher levels of TNF- $\alpha$ mRNA than normal skin after patch testing with SAg's $(P<0.05)$. Interestingly, TNF- $\alpha$ mRNA was markedly upregulated in the epidermis of SAg-induced skin at the 6-hour time point (Figure 5a), suggesting that keratinocytes were the initial targets for SEB-induced skin responses.

HLA-DR expression in tape-stripped skin. Significant MHC class II expression is not found on resting normal HKs, although treatment of HKs with cytokines such as IFN- $\gamma$ results in expression of this protein (25). HK MHC class II expression, however, is a feature of active psoriatic lesions that is reversed with standard psoriasis treatments including photochemotherapy (26). Because SAg's can act on keratinocytes through MHC class II molecules independent of T cells (9-11), we assessed HLA-DR expression by immunohistochemistry, in skin biopsies from psoriatics and control subjects, 6 hours after SEB or PBS application. As shown in Figure 7, psoriatic epidermis had significantly greater $(P<0.05)$ HLA-DR positivity than that from control subjects. In fact, 6 of 7 psoriatics and 0 of 6 normal patients expressed significant keratinocyte HLADR expression. Of note, specimens obtained from baseline uninvolved non-tape-stripped skin revealed 2 of 3 


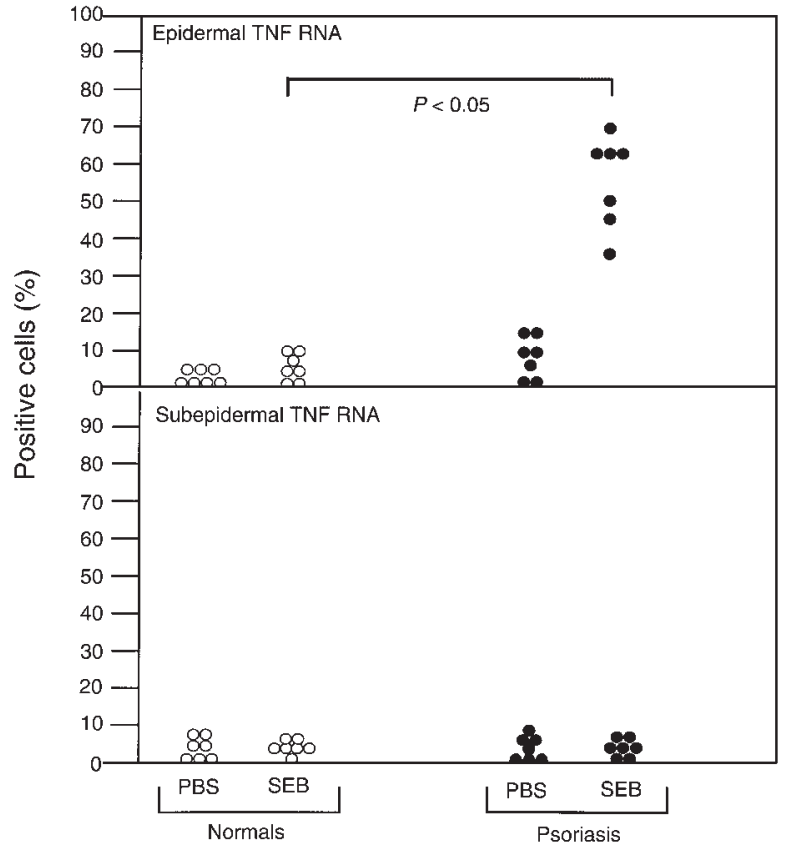

Figure 6

Expression of TNF- $\alpha$ mRNA in PBS- and SEB-stimulated skin from patients with psoriasis and from normal controls. As described in Methods, subjects underwent tape-stripping followed by application of either SEB or PBS vehicle for 6 hours, and tissue samples were subjected to in situ hybridization to measure TNF- $\alpha$ gene expression by in situ hybridization.

psoriatic, but 0 of 3 normal, subjects expressed a significant amount of epidermal HLA-DR. In the 2 psoriatic subjects who expressed significant amounts of HLA-DR ( $22 \%$ and $35 \%$ of epidermal cells positive), further increases were not seen after tape-stripping or SAg application. The 1 psoriatic patient who lacked HLADR at baseline had no induction of HLA-DR after tapestripping or SAg treatment. The epidermal MHC class II expression in representative psoriatic patients and control subjects after tape-stripping is pictured in Figure 5, c and d, respectively.

Skin reactivity to the G31S/S32P TSST-1 mutant protein. To determine whether SAg-induced skin inflammation in psoriasis was HLA-DR dependent, we examined the effects of a mutant TSST-1 protein, G31S/S32P, which does not bind HLA-DR, on uninvolved skin of patients with psoriasis by patch testing. In 5 psoriatic subjects tested, all 5 experienced positive inflammatory skin reactions to TSST $($ mean score $=1.4+$ ). However, the G31S/S32P TSST mutant protein did not elicit skin inflammation in any of these subjects, even when it was used at concentrations 10-fold higher than used for TSST-1 in Figure 2.

\section{Discussion}

Psoriasis is a common inflammatory autoimmune hyperproliferative skin disease that has a genetic component (reviewed in refs. 1-3, 27). The triggers that exacerbate psoriasis are important to identify, as they could become important therapeutic targets. Through their ability to act as SAg's, compelling evidence suggests streptococcal and staphylococcal exotoxins can initiate and/or propagate psoriasis in patients who are presumably genetically susceptible $(4,5,12)$. The ability of SAgstimulated immunocytes to induce psoriasis lesions in skin from psoriatic patients grafted to immunodeficient mice $(13,14)$ has provided a powerful experimental model system to study how these potent immune stimulators can initiate psoriasis. As proposed by Nickoloff and Wrone-Smith (28), T cells activated by SAg's could serve as initiators of the inflammatory events leading to psoriasis, with recruitment of autoreactive $T$ cells important in maintaining the psoriatic phenotype.

Given the accumulating evidence implicating SAg's in the initiation $(5,12)$ and propagation (4) of psoriasis, the current study examined the ability of topically applied staphylococcal and streptococcal exotoxins to induce cutaneous reactions. However, closed patch testing of $20 \mathrm{ng}$ of TSST-1, SEB, SPEA, and SPEC on intact skin failed to induce a clinical reaction in either psoriatic or normal subjects. Closed patch testing of much higher doses of SEB $\left(10 \mu \mathrm{g} / \mathrm{cm}^{2}\right.$ vs. $5 \mathrm{ng} / \mathrm{cm}^{2}$ used in this study) on the uninvolved skin of subjects with atopic dermatitis has been reported to induce cutaneous reactions (29), suggesting that our negative findings are probably due to the lower dosages of exotoxins used in this protocol.

One of the important clinical features associated with psoriasis is the presence of the Koebner (isomorphic) phenomenon, where traumatic insult to the skin is followed by new psoriasis lesions in nonlesional psoriatic skin $(30,31)$. Prospective studies indicate that cutaneous injuries will induce the Koebner phenomenon in $24-51 \%$ of patients and are increased when the disease is active (31). Repeated tape-stripping is included among the various types of trauma that can elicit the Koebner phenomenon $(31,32)$.

That psoriatics can respond to a host of traumatic insults with the initiation of new skin lesions suggested that epidermal barrier function perturbation may be required for development of cutaneous reactivity to low levels of exotoxins. Repeated tape-stripping of normal skin selectively removes the nonviable stratum corneum, which can allow enhanced penetration of many compounds (33). In addition, this procedure activates keratinocytes within hours, resulting in the upregulation of keratin-16 expression and both cytokine and adhesion molecule transcription (23). Because studies in normal patients indicate $T$ lymphocytes are not seen in tape-stripped skin for 24 hours (33), this early epidermal activation is believed to be due to resident epidermal cells. Cytokines reported to be induced by epidermal barrier disruption include TNF- $\alpha$, IFN- $\gamma$, IL-8, IL-10, and TGF- $\alpha(23,34)$. Similarly, ICAM-1 expression on keratinocytes is enhanced after tape-stripping (23).

Using tape-stripping to induce mild cutaneous modification, followed by closed patch testing of exotoxins, we observed cutaneous reactions at 48 hours. With both a clinical rating scale and direct measurement of 
mononuclear cell infiltrates, psoriatic subjects were found to experience a greater inflammatory skin response to SAg toxins than did normal controls. SEBinduced skin reactions demonstrated increased numbers of IFN- $\gamma$, but not IL- $4, \mathrm{mRNA}^{+}$activated $\mathrm{CD}^{+}$and $\mathrm{CD}^{+} \mathrm{T}$ cells. This increased reactivity to SAg's was not seen on unaffected skin of patients with active atopic dermatitis or lichen planus. Lichen planus is a nonhyperproliferative papulosquamous disease that was chosen because it is also characterized by an isomorphic phenomenon (35). The fact that the cutaneous reactivity of subjects with lichen planus was similar to that of normal or atopic subjects suggests that the hyperreactive cutaneous response we report in psoriatic skin is relatively selective for psoriasis.

Consistent with the clinical hyperreactivity noted in psoriatic subjects, in situ hybridization studies revealed increased TNF- $\alpha$ mRNA in the epidermis of psoriatic skin within 6 hours of SEB application. Our current finding of increased levels of TNF- $\alpha$ mRNA in vivo is consistent with previous reports that SAg's induce HK to produce TNF- $\alpha$ production in vitro $(10,24)$. Although selective expansion of T cells expressing specific TCR V $\beta$ types is a hallmark of SAg stimulation, immunohistochemical studies with a panel of antibodies to various TCR V $\beta$ types did not reveal increased expression of any one type of TCR V $\beta$ in the reactions created through this experimental protocol. These findings contrast with previous reports of $\mathrm{V} \beta$ skewing seen in actual lesions of acute guttate psoriasis $(5,12)$. The transient nature of lesions induced by epicutaneous application of exotoxins also differs from those of acute guttate psoriasis. One explanation for this dichotomy is that the exotoxins associated with guttate psoriasis are found on mucous membranes of the oropharynx, and release of toxin and/or selectively activated $\mathrm{T}$ cells initiates a psoriatic lesion when these activated T cells home to the skin. Of note, SAg-producing Streptococcus is not found on the skin of patients with guttate psoriasis. However, more than $50 \%$ of patients with plaque psoriasis do have toxin-producing $S$. aureus on their skin (2).

In contrast, the protocol used in this study evaluated the topical application of exotoxins on unaffected skin that not only has been activated, but made more permeable to epicutaneously applied agents due to loss of the stratum corneum. Since significant HLA-DR expression was found in 6 of 7 psoriatic but 0 of 6 normal subjects, we hypothesize that the SAg's interact directly with keratinocyte $\mathrm{MHC}$ class II proteins, resulting in keratinocyte cytokine production, with nonspecific Tcell recruitment as a secondary phenomenon. Our finding of HLA-DR expression in the uninvolved skin of psoriatics is consistent with published reports from other laboratories $(36,37)$. Although the skin lesions of lichen planus can express HLA-DR, there is no evidence for HLA-DR expression in unaffected skin of such patients $(38,39)$. Our current experiments using mutant TSST-1 protein provide direct support for this hypothesis. Thus, G31S/S32P, which does not bind the
HLA-DR molecule, did not induce an inflammatory skin repines, indicating that the exotoxin-induced skin changes were not due to a delayed hypersensitivity response to conventional antigen and that exotoxininduced skin inflammation proceeded from HLADR-mediated cell activation.

Taken together, these findings suggest an alternative mechanism in vivo by which SAg's may induce cutaneous inflammation in psoriasis. Because psoriatic patients are often colonized with Staphylococcus and Streptococcus (2), and scratching of the skin would be expected to mimic tape-stripping by both compromising the barrier function and activating the epidermis (23), this alternative mechanism may explain why some psoriatic patients report improvement of their disease activity after treatment with oral antibiotics (40). It may also account for several reports that fail to demonstrate selective expansion of TCR V $\beta$ s in the skin of patients with plaque psoriasis $(41,42)$. In this regard, epicutaneous application of SAg's on psoriatic skin may result in an acute inflammatory, HK-mediated response rather than SAg-mediated T-cell activation.

In addition to the ability of a SAg to interact with a HLA-DR-positive keratinocyte, psoriatics might show enhanced skin reactivity in this protocol because they have circulating activated lymphocytes that preferentially enter skin that is tape-stripped. Indeed, increased numbers of activated $T$ cells have been described in psoriatics (41). Of note, PBMCs from psoriatic patients have been reported to respond more vigorously to SEB and TSST- 1 in vitro than have normal control patient populations (43). As seen in the present study, this

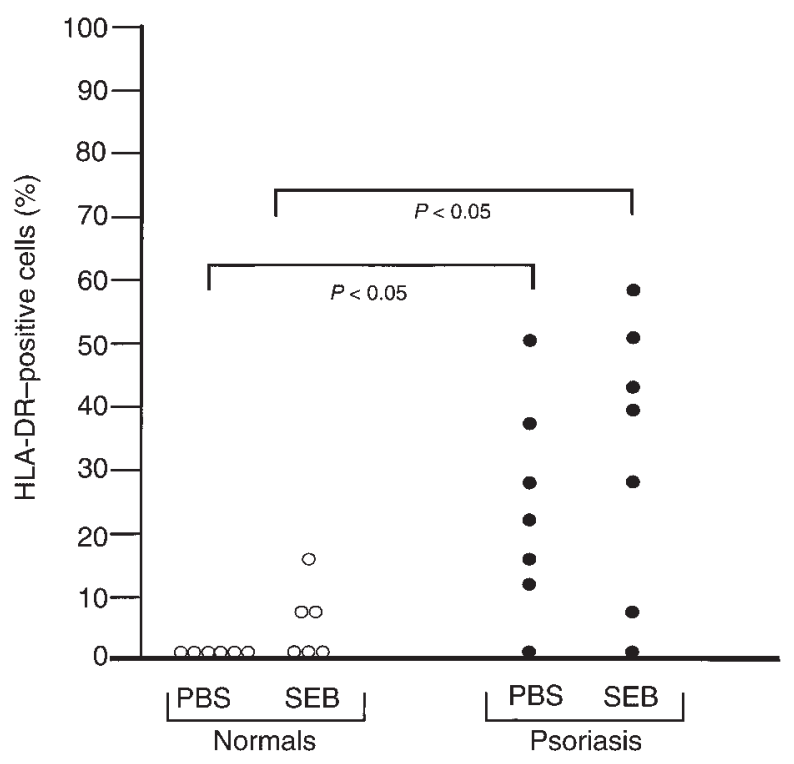

Figure 7

Expression of HLA-DR in PBS- and SEB-stimulated skin from patients with psoriasis and from normal controls. As described in Methods, subjects underwent tape-stripping followed by application of either SEB or PBS vehicle for 6 hours, and tissue samples were subjected to immunohistochemistry to assess HLA-DR protein expression. 
increased proliferative response to SAg's correlated with extent of disease activity (44). The increased cytokine gene and protein expression in psoriatic skin treated with SEB suggests an intrinsic psoriasis epidermal phenotype. This increased response to SEB and the early time course of response ( 6 hours) suggests an epidermal phenotype that favors stimulation in psoriasis.

As reported here and by Strange et al. (29), the ability to react to the epicutaneous application of exotoxins is not exclusive to psoriatics. However, the ability of traumatized skin to react to these SAg's appears to be more pronounced in subjects with psoriasis. As has been described with the Koebner phenomenon, this reactivity tends to correlate with disease activity (30, 31 ). Of note, 4 of the 5 psoriatic subjects who were found to have the highest cutaneous reactivity to the exotoxins also exhibited the Koebner phenomenon after skin biopsy. Taken together, these findings also suggest that epidermal modification is required for the optimal induction of SAg-induced skin inflammation in psoriasis. Epidermal modification results in the increased exposure of activated MHC class II-expressing keratinocytes to exotoxins; interaction of the SAg with the MHC class II-positive cells then results in the production of proinflammatory cytokines. The observation that atopic dermatitis skin reacted to SAg's in a manner similar to normal skin may be accounted for by the lack of HLA-DR expression on atopic skin (45).

The further characterization of this cutaneous hyperreactivity might provide exciting new directions for the future unraveling of the genetic and immunological mechanisms involved in psoriasis. Dissection of both genetic and immunological mechanisms involved in this common skin disease should have important consequences for its treatment.

\section{Acknowledgments}

Supported in part by research grants from the Public Health Services (HL-36577, AR-41256, HL-37260, AI31545, K081993, and HL-36611); a grant from the Division of Research Resources of the General Clinical Research Center (5 MO1 RR00051); and the University of Colorado Cancer Center. The authors thank Loren Golitz and John Aeling for technical assistance and histological interpretation of the cutaneous reactions; TsuYi Chuang for statistical help; and Maureen Sandoval for kind assistance in the preparation of this manuscript.

1. Nickoloff, B.J. 1991. The cytokine network in psoriasis. Arch. Dermatol. 127:871-884.

2. Leung, D.Y.M., et al. 1993. Presence of IgE antibodies to staphylococcal exotoxins on the skin of patients with atopic dermatitis. Evidence for a new group of allergens. J. Clin. Invest. 92:1374-1380.

3. Henderson, C.A., and Highet, A.S. 1988. Acute psoriasis associated with Lancefield Group C and Group G cutaneous streptococcal infections. Br. J. Dermatol. 118:559-561.

4. Leung, D.Y.M., Walsh, P., Giorno, R., and Norris, D.A. 1993. A potential role for superantigens in the pathogenesis of psoriasis. J. Invest. Dermatol. 100:225-228.

5. Leung, D.Y.M., et al. 1995. Evidence for a streptococcal superantigendriven process in acute guttate psoriasis. J. Clin. Invest. 96:2106-2112.

6. Kappler, J., et al. 1989. V beta-specific stimulation of human T cells by staphylococcal toxins. Science. 244:811-813.
7. Choi, Y.W., et al. 1989. Interaction of Staphylococcus aureus toxin "superantigens" with human T cells. Proc. Natl. Acad. Sci. USA. 86:8941-8945.

8. Kotzin, B.L., Leung, D.Y.M., Kappler, J., and Marrack, P. 1993. Superantigens and their potential role in human disease. Adv. Immunol. 54:99-166.

9. Nickoloff, B.J., et al. 1993. Accessory cell function of keratinocytes for superantigens. Dependence on lymphocyte function-associated antigen-1/intercellular adhesion molecule-1 interaction. J. Immunol. 150:2148-2159.

10. Tokura, Y., et al. 1994. Superantigenic staphylococcal exotoxins induce T-cell proliferation in the presence of Langerhans cells or class II-bearing keratinocytes and stimulate keratinocytes to produce T-cell-activating cytokines. J. Invest. Dermatol. 102:31-38.

11. Rich, R.R., Mollick, J.A., and Cook, R.G. 1989. Superantigens: interaction of staphylococcal enterotoxins with MHC class II molecules. Trans. Am. Clin. Climatol. Assoc. 101:195-204.

12. Lewis, H.M., et al. 1993. Restricted T-cell receptor $V$ beta gene usage in the skin of patients with guttate and chronic plaque psoriasis. Br.J. Dermatol. 129:514-520.

13. Boehncke, W.H., Dressel, D., Zollner, T.M., and Kaufmann, R. 1996. Pulling the trigger on psoriasis [letter]. Nature. 379:777.

14. Wrone-Smith, T., and Nickoloff, B.J. 1996. Dermal injection of immunocytes induces psoriasis. J. Clin. Invest. 98:1878-1887.

15. Henseler, T., and Christophers, E. 1985. Psoriasis of early and late onset: characterization of two types of psoriasis vulgaris. J. Am. Acad. Dermatol. 13:450-456.

16. Hanifin, J.M., and Rajka, G. 1980. Diagnostic features of atopic dermatitis. Acta Derm. Venereol. Suppl. (Stockh.) 92:44-47.

17. Blomster-Hautamaa, D.A., and Schlievert, P.M. 1988. Preparation of toxic shock syndrome toxin-1. Methods Enzymol. 165:37-43.

18. Murray, D.L., et al. 1996. Localization of biologically important regions on toxic shock syndrome toxin 1. Infect. Immun. 64:371-374.

19. Hurley, J.M., et al. 1995. Identification of class II major histocompatibility complex and $\mathrm{T}$ cell receptor binding sites in the superantigen toxic shock syndrome toxin 1. J. Exp. Med. 181:2229-2235.

20. Ying, S., et al. 1994. T lymphocytes and mast cells express messenger RNA for interleukin- 4 in the nasal mucosa in allergen-induced rhinitis. Immunology. 82:200-206.

21. Hamid, Q., Boguniewicz, M., and Leung, D.Y.M. 1994. Differential in situ cytokine gene expression in acute versus chronic atopic dermatitis. J. Clin. Invest. 94:870-876.

22. Frew, A.J., and Kay, A.B. 1988. The relationship between infiltrating CD4+ lymphocytes, activated eosinophils, and the magnitude of the allergen-induced late phase cutaneous reaction in man. J. Immunol. 141:4158-4164.

23. Nickoloff, B.J., and Naidu, Y. 1994. Perturbation of epidermal barrier function correlates with initiation of cytokine cascade in human skin.J. Am. Acad. Dermatol. 30:535-546.

24. Ezepchuk, Y.V., et al. 1996. Staphylococcal toxins and protein A differentially induce cytotoxicity and release of tumor necrosis factor-alpha from human keratinocytes. J. Invest. Dermatol. 107:603-609.

25. Wikner, N.E., et al. 1986. Study of HLA-DR synthesis in cultured human keratinocytes. J. Invest. Dermatol. 87:559-564.

26. Vallat, V.P., et al. 1994. PUVA bath therapy strongly suppresses immunological and epidermal activation in psoriasis: a possible cellular basis for remittive therapy. J. Exp. Med. 180:283-296.

27. Christophers, E., and Sterry, W. 1993. Psoriasis. In Dermatology in general medicine. T.B. Fitzpatrick, A.Z. Eisen, K. Wolff, and K.F. Austen, editors. McGraw-Hill, New York, NY. 489-514.

28. Nickoloff, B.J., and Wrone-Smith, T. 1998. Superantigens, autoantigens, and pathogenic T cells in psoriasis [letter]. J. Invest. Dermatol. 110:459-460.

29. Strange, P., Skov, L., Lisby, S., Nielsen, P.L., and Baadsgaard, O. 1996. Staphylococcal enterotoxin B applied on intact normal and intact atopic skin induces dermatitis. Arch. Dermatol. 132:27-33.

30. Koebner, J. 1877. Zur aetiologie der psoriasis. Vjscher. Derm. 4:203-207.

31. Eddy, D., Ascheim, E., and Farber, E.M. 1964. Experimental analysis of isomorphic (Koebner) response in psoriasis. Arch. Dermatol. 89:579-588.

32. Heng, M.C., Kloss, S.G., Kuehn, C.S., and Chase, D.G. 1985. The sequence of events in psoriatic plaque formation after tape-stripping. $\mathrm{Br}$. J. Dermatol. 112:517-532.

33. Pinkus, H. 1951. Examination of epidermis by the strip method II. Biometric data on regeneration of the human epidermis. J. Invest. Dermatol. 19:431-447

34. Wood, L.C., Jackson, S.M., Elias, P.M., Grunfeld, C., and Feingold, K.R. 1992. Cutaneous barrier perturbation stimulates cytokine production in the epidermis of mice. J. Clin. Invest. 90:482-487.

35. Arndt, K. 1993. Lichen planus. In Dermatology in general medicine. T.B. Fitzpatrick, A.Z. Eisen, K. Wolff, and K.F. Austen, editors. McGraw-Hill. New York, NY. 1134-1144.

36. Esgleyes-Ribot, T., Chandraratna, R.A., Lew-Kaya, D.A., Sefton, J., and Duvic, M. 1994. Response of psoriasis to a new topical retinoid, AGN 190168. J. Am. Acad. Dermatol. 30:581-590. 
37. Paukkonen, K., Naukkarinen, A., and Horsmanheimo, M. 1995. The development of manifest psoriatic lesions is linked with the appearance of ICAM-1 positivity on keratinocytes. Arch. Dermatol. Res. 287:165-170.

38. Farthing, P.M., Matear, P., and Cruchley, A.T. 1992. Langerhans cell distribution and keratinocyte expression of HLADR in oral lichen planus. J. Oral Pathol. Med. 21:451-455.

39. Volc-Platzer, B., Groh, V., and Wolff, K. 1987. Differential expression of class II alloantigens by keratinocytes in disease. J. Invest. Dermatol. 89:64-68.

40. Rosenberg, E.W., et al. 1986. Use of rifampin with penicillin and erythromycin in the treatment of psoriasis. Preliminary report. J. Am. Acad. Dermatol. 14:761-764.

41. Schmitt-Egenolf, M., Boehncke, W.H., Christophers, E., Stander, M., and Sterry, W. 1991. Type I and type II psoriasis show a similar usage of T- cell receptor variable regions. J. Invest. Dermatol. 97:1053-1056.

42. Boehncke, W.H., et al. 1995. T-cell-receptor repertoire in chronic plaquestage psoriasis is restricted and lacks enrichment of superantigen-associated V beta regions. J. Invest. Dermatol. 104:725-728.

43. Jeffes, E.W., III, et al. 1995. Elevated numbers of proliferating mononuclear cells in the peripheral blood of psoriatic patients correlate with disease severity. J. Invest. Dermatol. 105:733-738.

44. Yokote, R., Tokura, Y., Furukawa, F., and Takigawa, M. 1995. Susceptible responsiveness to bacterial superantigens in peripheral blood mononuclear cells from patients with psoriasis. Arch. Dermatol. Res. 287:443-447.

45. Barker, J.N., and MacDonald, D.M. 1987. Epidermal class II human lymphocyte antigen expression in atopic dermatitis: a comparison with experimental allergic contact dermatitis. J. Am. Acad. Dermatol. 16:1175-1179. 